Military Technical College Kobry El-Kobbah, Cairo, Egypt.

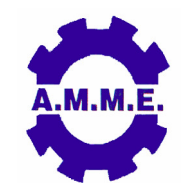

$15^{\text {th }}$ International Conference on Applied Mechanics and Mechanical Engineering.

\title{
COMPUTER AIDED MANUFACTURING IN FIVE AXES FOR STATOR BLADES
}

\author{
A. But ${ }^{*}$
}

\section{ABSTRACT}

In this paper, the computer aided manufacturing solution is presented for manufacturing a complex surface of the stator blades in addition to the technology implementation on the CNC machine tools in five axes. The particularities of the ESPRIT CAM software are underlining. In the present work, the last module created by DP Technology (dedicate to manufacturing the impeller) is used. Starting with the import of the CAD model, the technology is elaborated and the NC program is generated. The optimized solution, using two different milling strategies, is underlining the capabilities of the CAM software.

\section{KEY WORDS}

CAM application, CNC machine tools, stator blades.

\footnotetext{
* Lecturer, Dept. of Materials and Manufacturing Engineering, Mechanical Faculty,
} "POLITEHNICA" University of Timisoara, Romania. 


\section{INTRODUCTION}

The three-dimensional geometry of runner blade has an important influence on the hydraulic performances. For example, a very small design modification of blade profiles, both near blade leading edge and near trailing edge, has a crucial effect on upper part load pressure pulsations.

During the aerodynamic research of turbo machinery, the Design of Experiments (DOE) technique and the Response Surface Methodology (RSM) are widely used to optimize the turbo machinery's performance [1-2].

In this paper, a real application which represents the research subject to manufacturing the turbine blades is presented. The solution to generate the complex parts on CNC machine tool is also presented. The CAD model imported for manufacturing was projected in the Machine in the Hydraulic Department, "Politehnica", University of Timisoara, Romania, Cf. Fig.1. This subassembly is part of an experimental setup which is used in the laboratory to analyze the development; implementation and testing the new flow control techniques in which rotation of the liquid jet that does not introduce volume loss in the system and requires no additional energy input.

Rotating flows have an important role in industrial applications (burners, turbines and hydraulic pumps, etc.). Aero/hydro- knowledge flow rotation allows development and testing of hydro-dynamic flow methods tested in the laboratory [3]. Figure 2 shows the rotating flow effect that intended to be obtained with the experimental stand.

The stator (the guide vane) generates a free vortex flow with constant rotation, and the axial force generates a constant pressure [3]. The manufacturing solution of this part is the subject of the article.

\section{METHODOLOGY}

In this paper, a real application is presented which represents the research subject to manufacturing a turbine blade. The technological solutions was analyzed and presented an optimize solution. The most interest facts were: the time of manufacturing and the cost of the implementation. The technological steps taken into consideration when manufacturing the turbine blade are:

$\downarrow$ Roughing between blades

$\downarrow$ Hub Rough

$\downarrow$ Hub Finishing

$\downarrow$ Blade Finishing

$\downarrow$ Fillet finishing

The CAD model-generated with CATIA v5R16 CAD software, which was imported 
for modelling the stator blades is shown in Fig. 3. The numeric analyses have been done using "FLUENT 6.3" software.

To manufacture the turbine blades, Fig 3, it will be necessary to use CNC machine tools in five axes (Fig 4). To generate the NC program for the milling center, ESPRIT CAM software is used. The first step is to "Setup the Machine Tool" -to define correctly what are the linear moving and what are the rotations moving and what parts of the machine tools will generate all this.

The models of CNC machine tool in five axes can be: a) horizontal or vertical milling center with "trunnion"-the two rotations are generated by the table, b) machine tool in five axes which can generate the rotations moving by the head, c) the mill-turn center where the axis B ( rotation ) is generated by the head and the axis $C$ (the second rotation) represents one rotation around the $\mathrm{Z}$ axis and $\mathrm{d}$ ) another model with five axes machine tool which can generate one rotation $360^{\circ}$ by the table and another rotation by the head (the model which was used in our application with the head rotation possibilities between $-90^{\circ}$ and $+90^{\circ}$ ) [5], Cf. Fig. 4 .

\section{Roughing between blades}

Rough paths need to start outside of the material. Paths should be extended to start and end outside of the area between 2 blades. For our concrete example, Fig. 3, first we must make "Outer Contouring" as shown in Fig. 5, to prepare the geometry of the raw part to be able to manufacturing the blade.

For this operation, one cutting tool "end mill" with $32 \mathrm{~mm}$ diameter is used and the cutting tool parameters are: cutting speed $=500 \mathrm{~m} / \mathrm{min}, \mathrm{n}=4974 \mathrm{rot} / \mathrm{min}$, feeding rate $=2984 \mathrm{~mm} / \mathrm{min}$ and $\mathrm{fz}=0.2$. The raw part is CuZn35Mn2FeAINi, a cupper alloy material, dedicated to work in fresh water and sea.

Another preparation operation is "Upper Groove" and "Lower Groove" shown in Fig. 6. With these two technological operations, the possibilities to enter and generate the surface are created for of every blade. This operation was manufacturing with one cutting tool "end mill" with $25 \mathrm{~mm}$ diameter.

Axis of the machine tools can be able to generate the complex surfaces. For this operation, one cutting tool " end mill " with $25 \mathrm{~mm}$ diameter is used and the cutting tool parameters are: cutting speed $=500 \mathrm{~m} / \mathrm{min}, \mathrm{n}=6366 \mathrm{rot} / \mathrm{min}$, feeding rate $=1909$ $\mathrm{mm} / \mathrm{min}$, and $\mathrm{f} z=0.1$.

\section{Hub Rough}

Rough paths need to start outside of the material. Passes should be extended to start and end outside the area between 2 blades. Extending paths could be done in ESPRIT by extending the 2 guide ruled features.

With this operation, each turbine blade was generated separately. This operation should be copied 12 times more around $z$ axis in order to be repeated for each 
blade. For this operation, one "end mill" cutting tool with $12 \mathrm{~mm}$ diameter is used, Cf. Fig. 7. This operation needs all five axes of the machine tools to be able of generating the complex surfaces.

For this operation, one cutting tool "end mill "with $12 \mathrm{~mm}$ diameter is used and the cutting tool parameters are: cutting speed $=350 \mathrm{~m} / \mathrm{min}, \mathrm{n}=9284 \mathrm{rot} / \mathrm{min}$, feeding rate $=4999 \mathrm{~mm} / \mathrm{min}$ and $\mathrm{f} z=0.18$.

Using face profile, 4 feature chains will be created for top and bottom of each channel side. Now, the order of priority is the selection of machining profile. All 4 chains MUST be oriented the same way going from trailing edge to leading edge of the blade. Ruled features from wire frame geometry will be created and these features MUST follow the direction of the stator axis.

Free Form feature will be created with Hub and fillet as part, and the fillet faces will be selected with along parametric face propagation. For manufacturing the CAM software have more options and for our concrete Model, the following are selected:

- Roughing Direction: Bottom Up (Fig. 8b)

- Machining Pattern: Parametric

- Cutting Direction: U (Fig. 8a)

\section{Hub Finishing}

The rest material remains on the stator hub, so it is necessary to manufacture such a surface. For this operation, a small cutting tool, a "ball nose" by $6 \mathrm{~mm}$ diameter is used (Fig. 9). The surface limitation between two blades and the trajectory for manufacturing by the cutting tool are selected.

For this operation, one cutting tool "end mill "with $6 \mathrm{~mm}$ diameter is used and the cutting tool parameters are: cutting speed $=300 \mathrm{~m} / \mathrm{min}, \mathrm{n}=15915 \mathrm{rot} / \mathrm{min}$, feeding rate $=1591 \mathrm{~mm} / \mathrm{min}$ and $\mathrm{fz}=0.05$.

\section{Blade Finishing}

The tehnological operation applied herein using different milling strategies in order to improve cycle time of manufacturing. The same feeding and cutting speed for both milling strategies were used. First, the blade finishing was tested with "Project Parametric Pattern" strategy, and the second milling strategy was tested with "Project Spiral" milling strategy.

For this operation, one cutting tool "end mill" with $8 \mathrm{~mm}$ diameter is used and the cutting tool parameters was: cutting speed $=450 \mathrm{~m} / \mathrm{min}, \mathrm{n}=17905 \mathrm{rot} / \mathrm{min}$, feeding rate $=2148 \mathrm{~mm} / \mathrm{min}$ and $\mathrm{fz}=0.06$.

In this paper, the values of the cutting tools parameters are presented. These values are calculated by the CAM software and used to manufacturing the part. These high 
values of the parameters were able to be implemented by using the special coating of the SANDVIK cutting plates, and realized by the high constructive characteristics of the machine tools.

\section{Fillet finishing}

The last step is finishing the fillet at the connection of the blade and the hub. The strategy of: "composite cycle with project spiral pattern and orientation through profile" was applied.

The difficulty of this operation is the tool orientation. Through profile orientation often offers the most flexibility. Best orientation curve is an open curve for blades.

"Snap mode", is created by a series of segments that are connected on both sides as shown in Fig. 11. Then, the middle points of the segments are connected. The lines design the final form will be generated.

\section{CONCLUSION}

ESPRIT offers the possibility to chose, change the blade turbine manufacturing strategies in five axes. It is very important to choose the correct strategy in order to obtain the best results [4]. In this case, the Blade Finishing with Project Parametric Pattern Tool Path strategy is chosen for simulating process in five axes.

Using ESPRIT, we can obtain one report for each strategy.

Simulation processing in five axes:

\section{FIRST SOLUTION}

Blade Finishing with Project Parametric

Pattern

Time for:

Outer Contouring,

Upper Groove,

Lower Groove 1,

Lower Groove 2,

Hub Rough,

Hub Finishing,

Blade Finishing

Parametric Pattern,

\section{SECOND SOLUTION}

Blade Finishing with Project Spiral

Time for:

Outer Contouring, $\quad$ 00:01:24

Upper Groove, $\quad$ 00:00:17

Lower Groove 1, 00:03:04

Lower Groove 2, $\quad$ 00:01:43

Hub Rough, $\quad$ 00:00:41

Hub Finishing 00:04:32

Blade Finishing

with Project Spiral,

The effective time processing for the first solution is $23 \mathrm{~min} 09 \mathrm{sec}$, whereas the effective time processing for the second solution is $21 \mathrm{~min} 8 \mathrm{sec}$. The difference time 
is $1 \mathrm{~min} 29 \mathrm{sec}$. the total time for manufacturing all blades turbine with 13 blades is $15 \mathrm{~min} 48 \mathrm{sec}$.

In Fig. 12, the total time for manufacturing on five axes milling centre, for the turbine blade, using the "Parametric Pattern" strategy (12 min $48 \mathrm{sec}$ ), is presented in comparison with the manufacturing time applied with the second milling strategy "Spiral blade finishing" ( $11 \mathrm{~min} 19 \mathrm{sec}$ ). If the total time for manufacturing one piece of turbine blade with 13 blades will be counted, one reduction time with $18 \mathrm{~min}$ and $17 \mathrm{sec}$ will be obtained which represents a growing productivity with $11 \%$.

Advantage: The main advantage of five-axes machining is the ability to save time by machining of complex shapes in a single set-up. Another benefit comes from allowing the use of shorter cutters that will permit more accurate machining. Using a $\mathrm{CNC}$ machine-tool in five axes, we can grow up the accuracy and will be reduced the operation time.

\section{REFERENCES}

[1] Bonaiuti D., Arnone A., and Ermini M., "Analysis and Optimization of Transonic Centrifugal Compressor Impellers Using the Design of Experiments Technique ", ASME J. of Turbo machinery, 128(4) 786-797 (2006).

[2] Bonaiuti D. and Zangeneh M., "On the Coupling of Inverse Design and Optimization Techniques for the Multiobjective, Multipoint Design of Turbo machinery Blades", ASME J. of Turbo machinery, 131(2), (2009).

[3] Ciocan G. D. and Iliescu M. S., "Vortex Rope Investigation by 3D -PIV Method Production" of the 2 IAHR International Meeting of the Workgroup on Cavitations and Dynamic Problems in Hydraulic Machinery and Systems, Timisoara, Romania (2007).

[4] Scientific Bulletin of the "Polytechnic" University of Timisoara -Transaction on Mechanics, Vol. 52(66), Issue 6, pp. 159-177

[5] But A., "The influence of CNC lathe construction on manufacturing accuracy." Editura POLITEHNICA-Timisoara (2003).

[6] But A., "Machine tolos and avance systems of manufacturing". Editura POLITEHNICA-Timisoara (2009). 

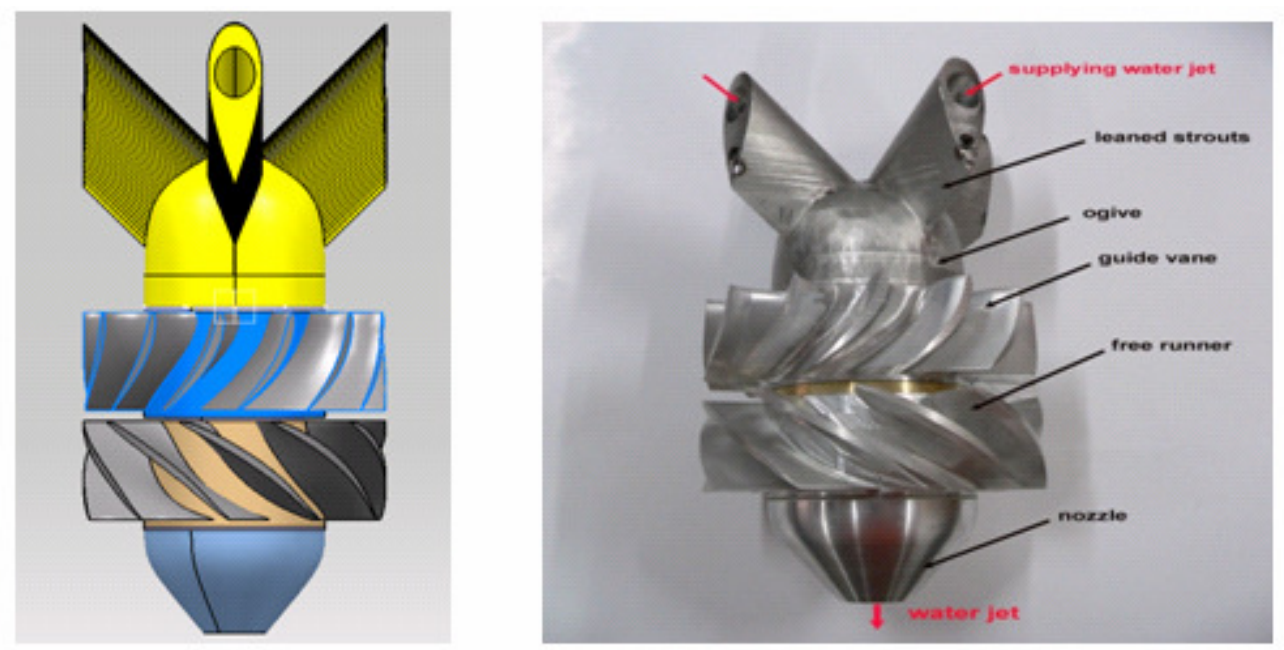

Fig.1. The subassembly which includes the stator blade (guide vane). The CAD and the real model designed and executed.
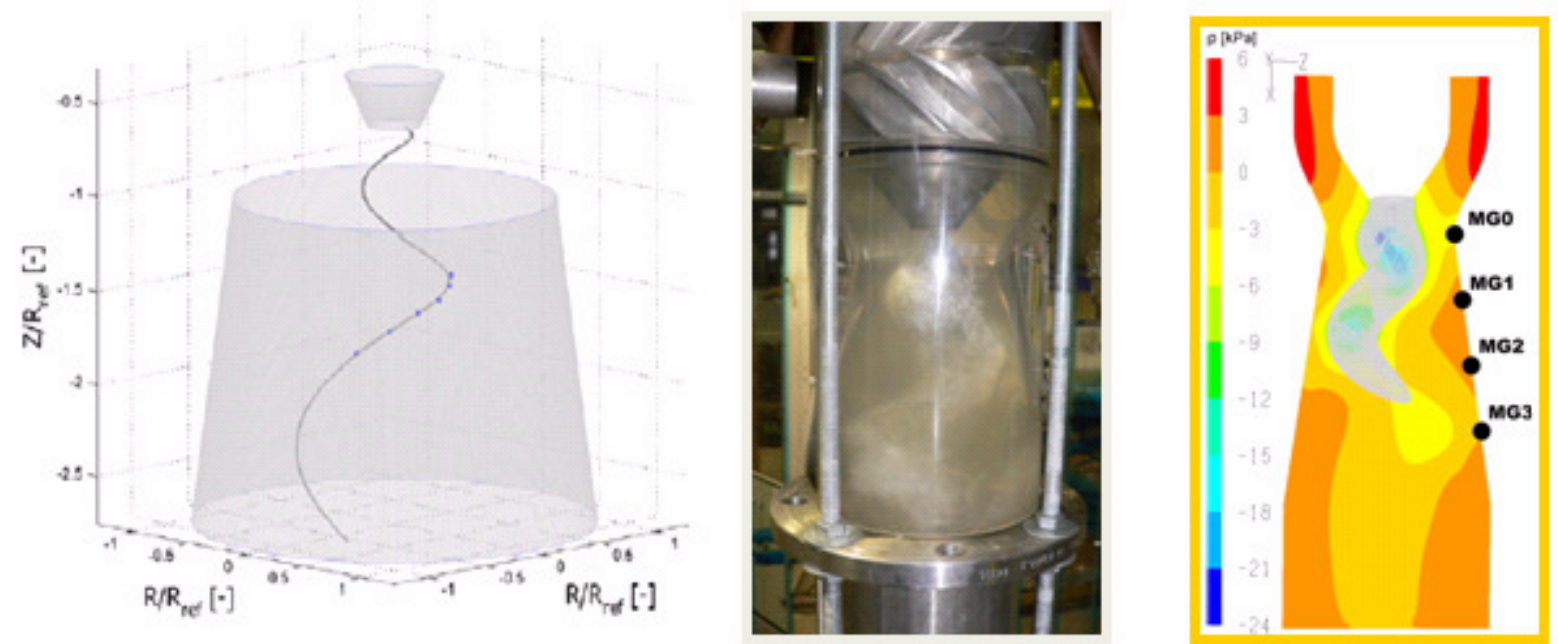

Fig.2. The rotation flow effect that was intended to be obtained with the experimental stand 


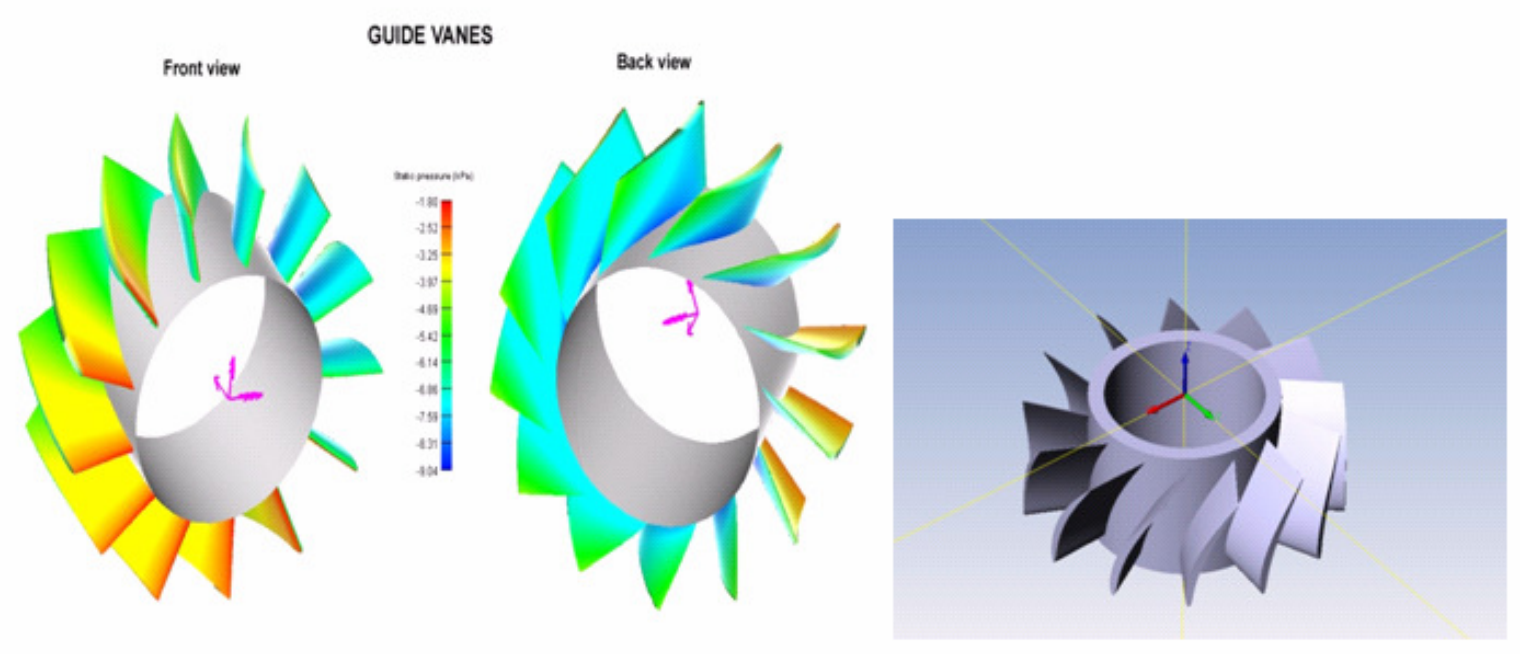

Fig. 3. The CAD model of the stator blades.

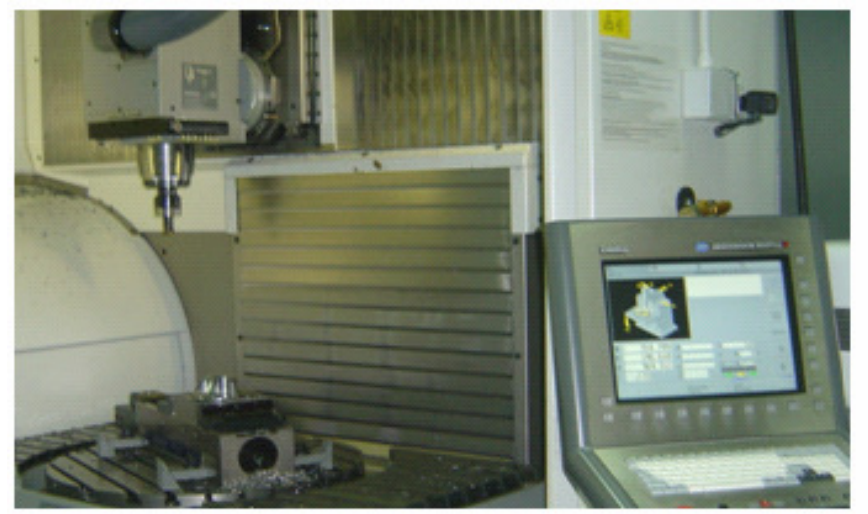

Fig. 4. Five axes Milling centres with one rotations made by the table $\left(360^{\circ}\right)$, and another rotation between $-90^{\circ} /+90^{\circ}$ made by head.

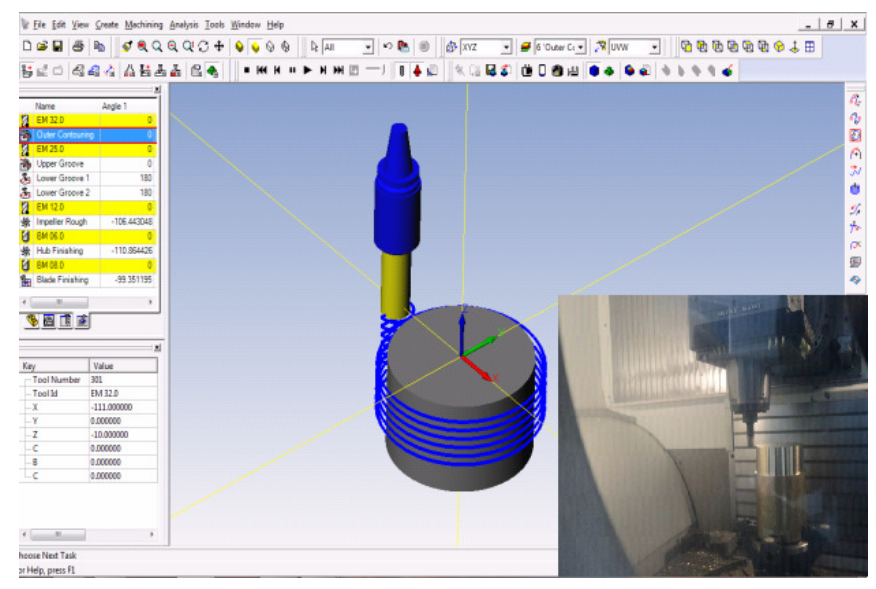

Fig. 5. The "Outer Contouring" milling operation. 


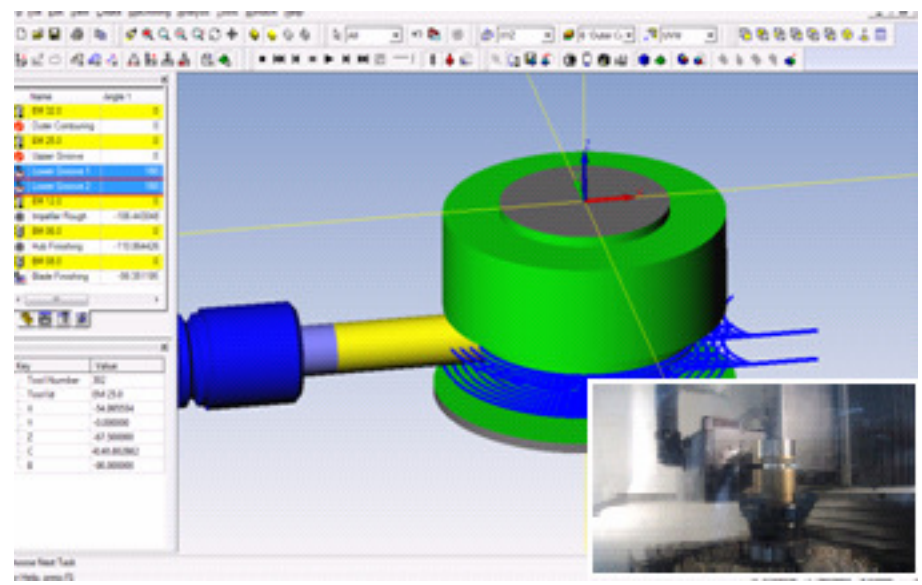

Fig. 6. The "Lower Groove" operations.

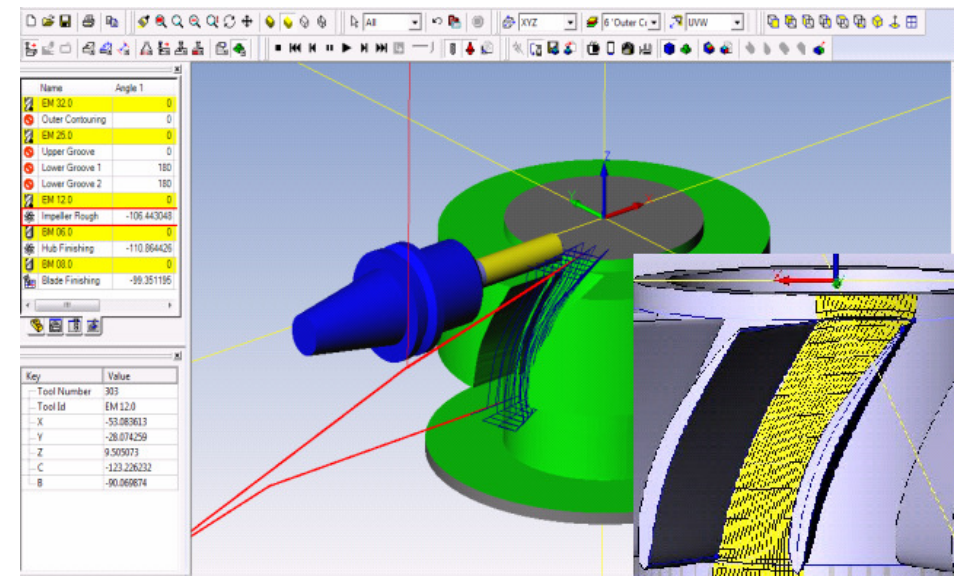

Fig. 7. The Impeller milling Rough operation.

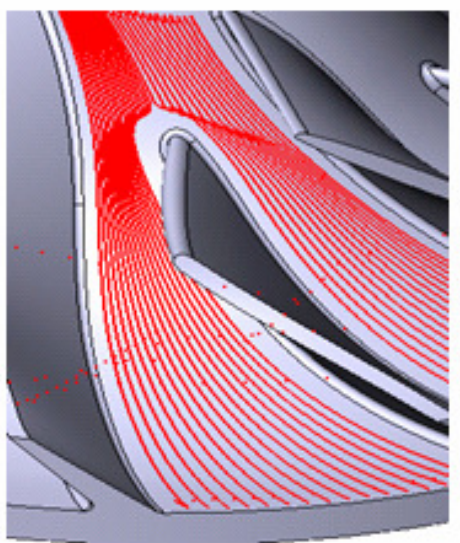

a)

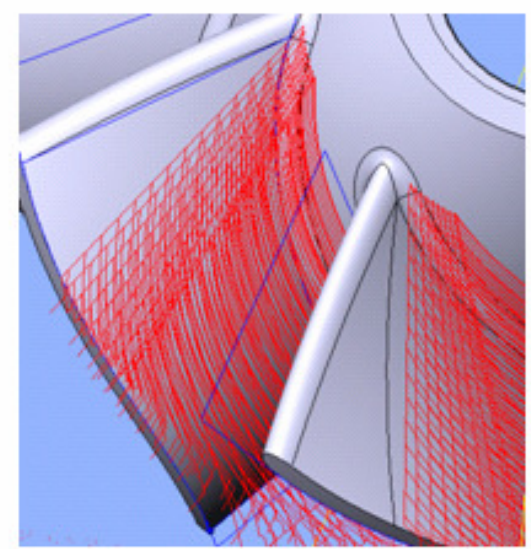

b)

Fig. 8. Manufacturing strategies :a)Cutting Direction: $U, b)$ Roughing Direction: Bottom Up 


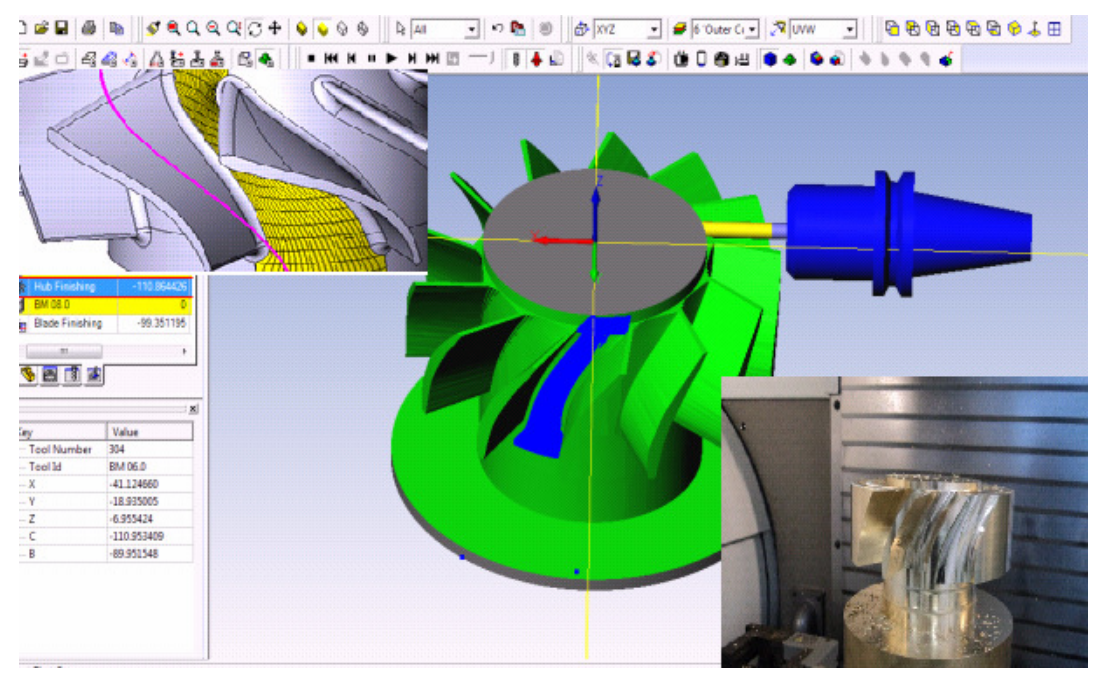

Fig. 9. The stator hub finishing operation.

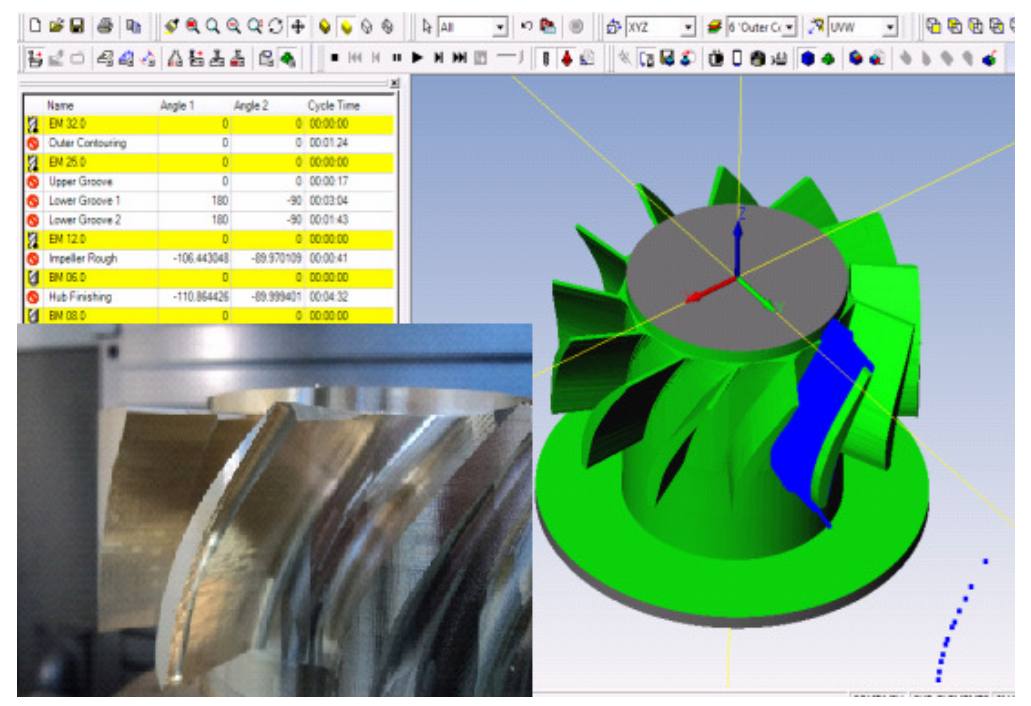

Fig. 10. The Impeller Blade Finishing operation.
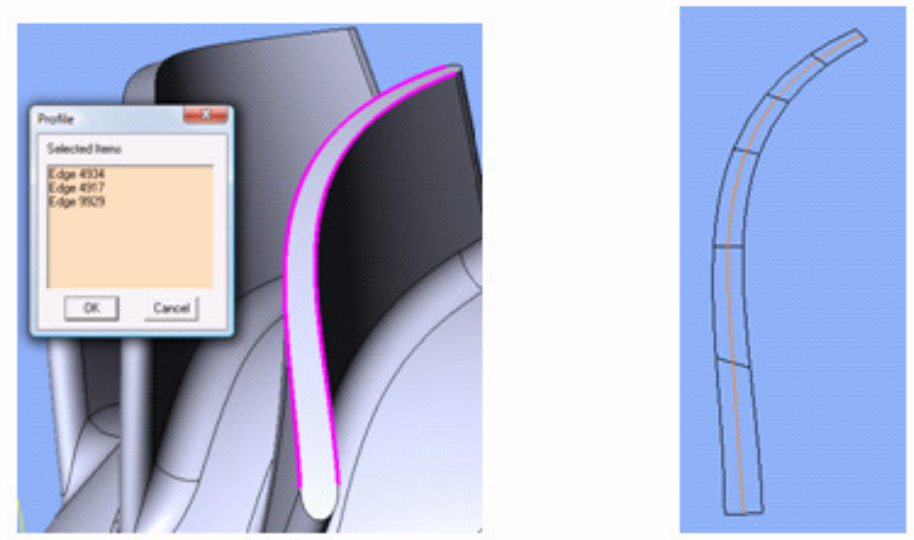

Fig. 11. Modality to create the tool trajectory for fillet finishing 


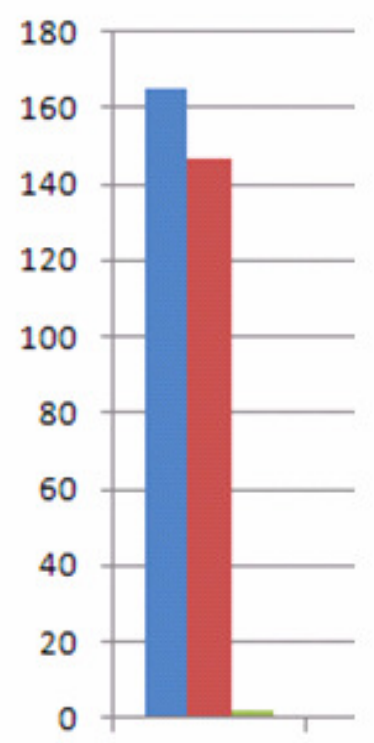

Blade Finishing with Project Parametric Pattern165min $12 \mathrm{sec}$ a Finishing with Project Spiral $146 \mathrm{~min} 50 \mathrm{sec}$

Fig. 12. Processing time diagram for the two strategies used 